\title{
Correction to: Report of the IAU Working Group on Cartographic Coordinates and Rotational Elements: 2015
}

\author{
B. A. Archinal ${ }^{1}$ D . C. H. Acton ${ }^{2}$ - A. Conrad ${ }^{3} \cdot$ T. Duxbury $^{4}$ - D. Hestroffer ${ }^{5}$ - J. L. Hilton ${ }^{6}$. \\ L. Jorda ${ }^{7} \cdot$ R. L. Kirk ${ }^{1} \cdot$ S. A. Klioner ${ }^{8} \cdot$ J.-L. Margot ${ }^{9} \cdot$ K. Meech ${ }^{10} \cdot$ J. Oberst $^{11}$. \\ F. Paganelli ${ }^{12}$. J. Ping ${ }^{13}$ - P. K. Seidelmann ${ }^{14}$. A. Stark ${ }^{11}$ - D. J. Tholen ${ }^{15} \cdot$ Y. Wang $^{16}$. \\ I. P. Williams ${ }^{17}$
}

Published online: 2 December 2019

(c) Springer Nature B.V. 2019

\section{Correction to: Celest Mech Dyn Ast (2018) 130:22 https://doi.org/10.1007/s10569-017-9805-5}

\begin{abstract}
We point out some errors in the most recent report from the International Astronomical Union (IAU) Working Group on Cartographic Coordinates and Rotational Elements (Archinal
\end{abstract}

The original article can be found online at https://oi.org/10.1007/s10569-017-9805-5.

B. A. Archinal

barchinal@usgs.gov

Astrogeology Science Center, U.S. Geological Survey, Flagstaff, AZ, USA

Jet Propulsion Laboratory, Pasadena, CA, USA

3 Large Binocular Telescope Observatory, University of Arizona, Tucson, AZ, USA

4 George Mason University, Fairfax, VA, USA

5 IMCCE, Observatoire de Paris, PSL Research University, CNRS, Sorbonne Universités, UPMC, Univ. Lille, Paris, France

6 U.S. Naval Observatory, Washington, D.C, USA

7 Laboratoire d'Astrophysique de Marseille, Marseille, France

8 Lohrmann Observatory, Technische Universtät Dresden, Dresden, Germany

9 University of California, Los Angeles, CA, USA

10 Institute for Astronomy, Honolulu, HI, USA

11 German Aerospace Center, DLR, Berlin, Germany

12 SETI Institute, Mountain View, CA, USA

13 National Astronomical Observatories of CAS, Beijing, China

14 University of Virginia, Charlottesville, VA, USA

15 University of Hawaii, Honolulu, HI, USA

16 School of Astronautics, Beihang University, Beijing, China

17 Queen Mary, University of London, London, UK 
et al. 2018). We correct a sign error in Figs. 1 and 2. We also correct the equation for the prime meridian position $(W)$ of Mars' satellite Phobos in Table 2.

Two separate errors have been made. First, in Figs. 1 and 2 of Archinal et al. (2018), the expression giving the right ascension of the node $Q$ incorrectly has a negative sign. It should read " $90^{\circ}+\alpha_{0}$ ". The corrected versions of both figures are shown below along with the original captions, which were not affected by the error.

Second, there are two mistakes in the equation for the prime meridian position $(W)$ of Mars' satellite Phobos reported in Table 2 of the report. This equation is based on the derivation by Stark et al. (2017) but with a correction to the prime meridian constant $W_{0}$ due to the forced libration in longitude, i.e. based on equations 3 and 10 of Burmeister et al. (2018). However, at present the prime meridian of Phobos is defined dynamically, i.e. the prime meridian is defined by the orientation of Phobos with respect to Mars center of

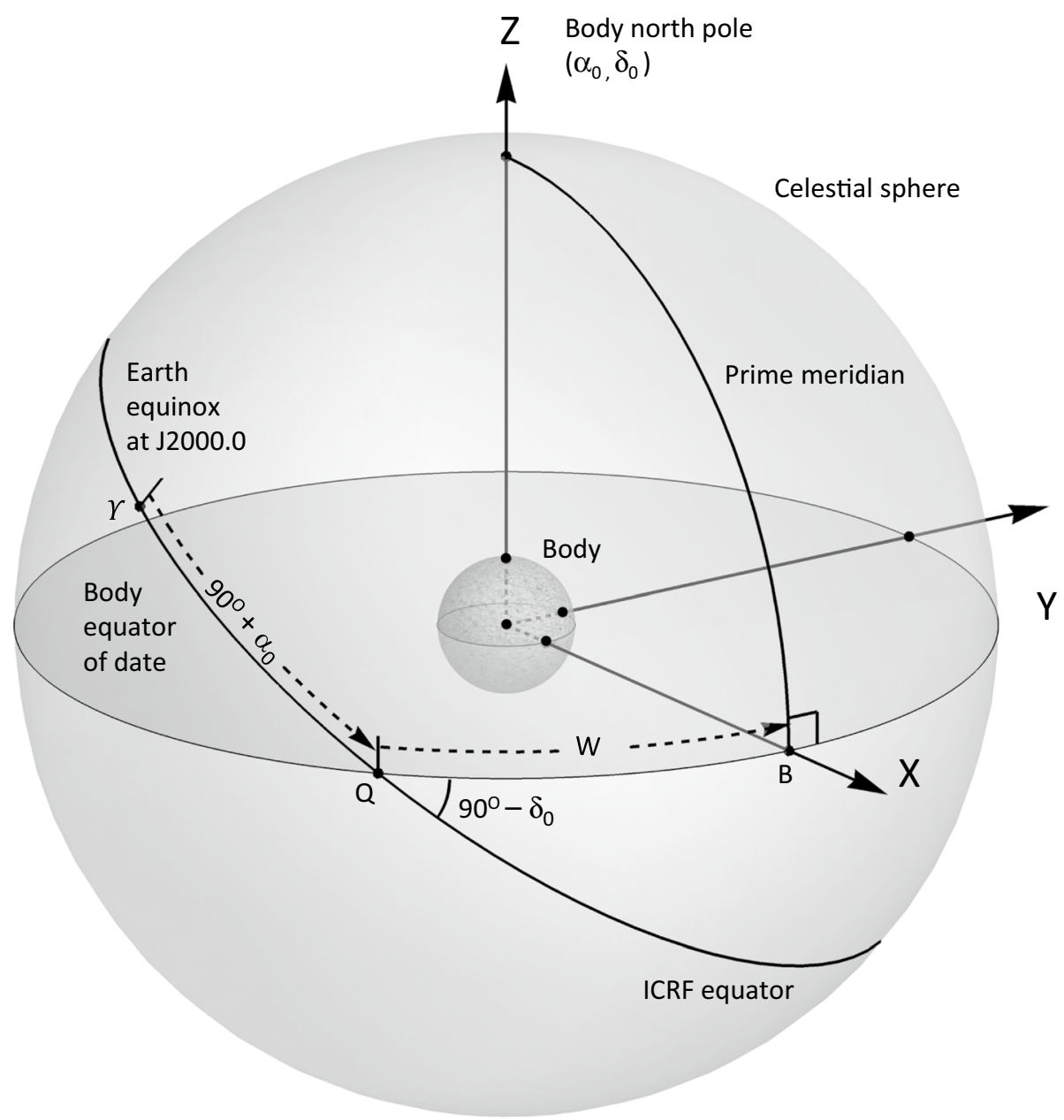

Fig. 1 Reference system used to define orientation of the planets and their satellites. For $W(t)>0$, body rotation is prograde (e.g. Mercury, Jupiter). For $W(t)<0$, body rotation is retrograde (e.g. Venus, Uranus) 


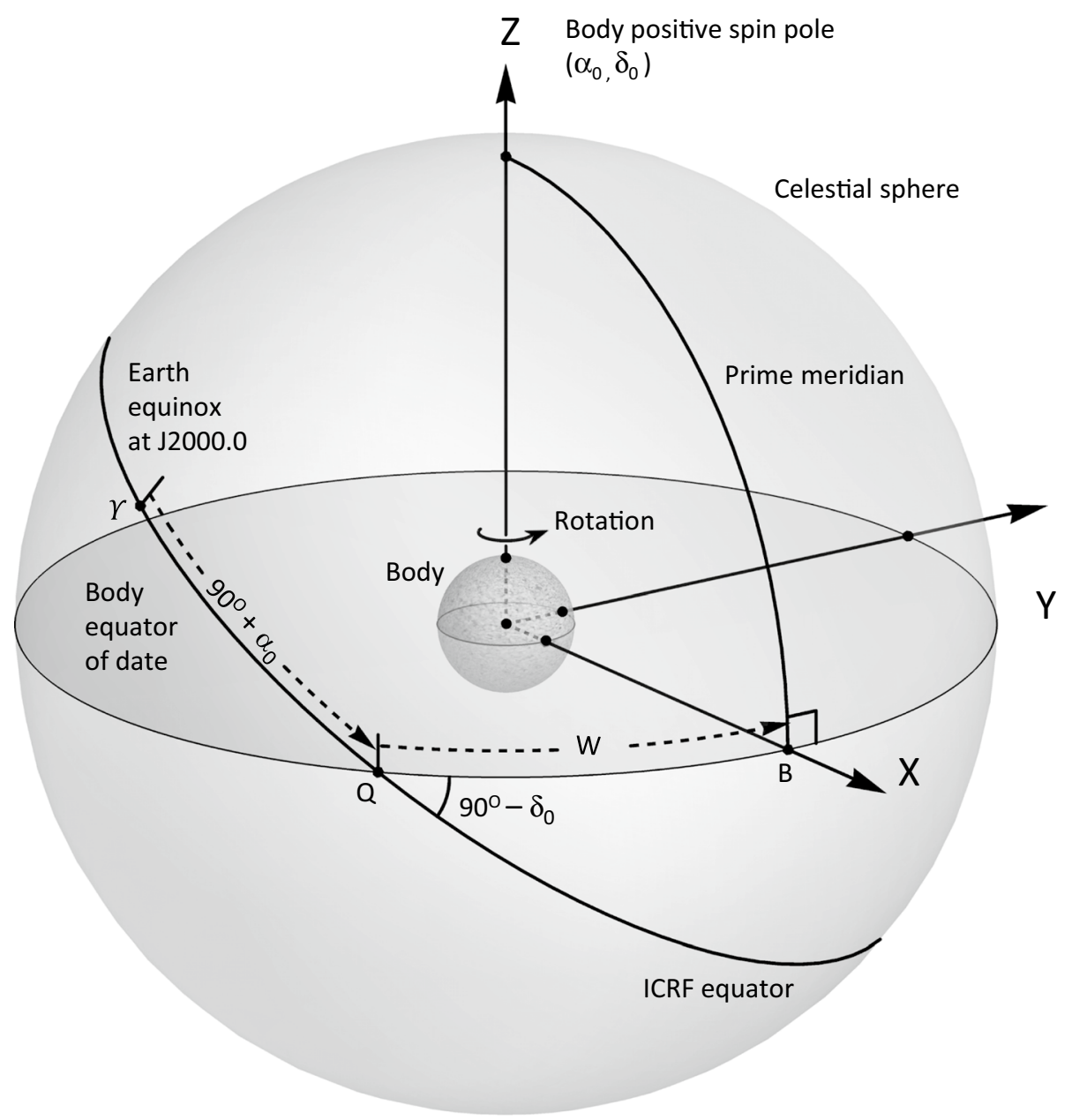

Fig. 2 Reference system used to define the orientation of dwarf planets, minor planets, their satellites, and comets

mass at the time the satellite is passing its pericenter. To maintain this definition, the correction term for $W_{0}$ introduced by Burmeister et al. [2018; “ $p_{\lambda} \sin \left(189.6327156^{\circ}\right)$ "] is not appropriate and the value of the prime meridian constant (first term in the equation for $W$ ) should have read " 35.18774440 ". In addition, the sign of the last term ["1.143 $\sin (M 5)$ "] was incorrectly shown as positive and should have been negative.

We have reproduced the corresponding portion of Table 2 below, correcting the equation for $W$, and including the full set of equations for the orientation of Phobos. 
Table 2 Recommended values for the direction of the north pole of rotation and the prime meridian of the satellites

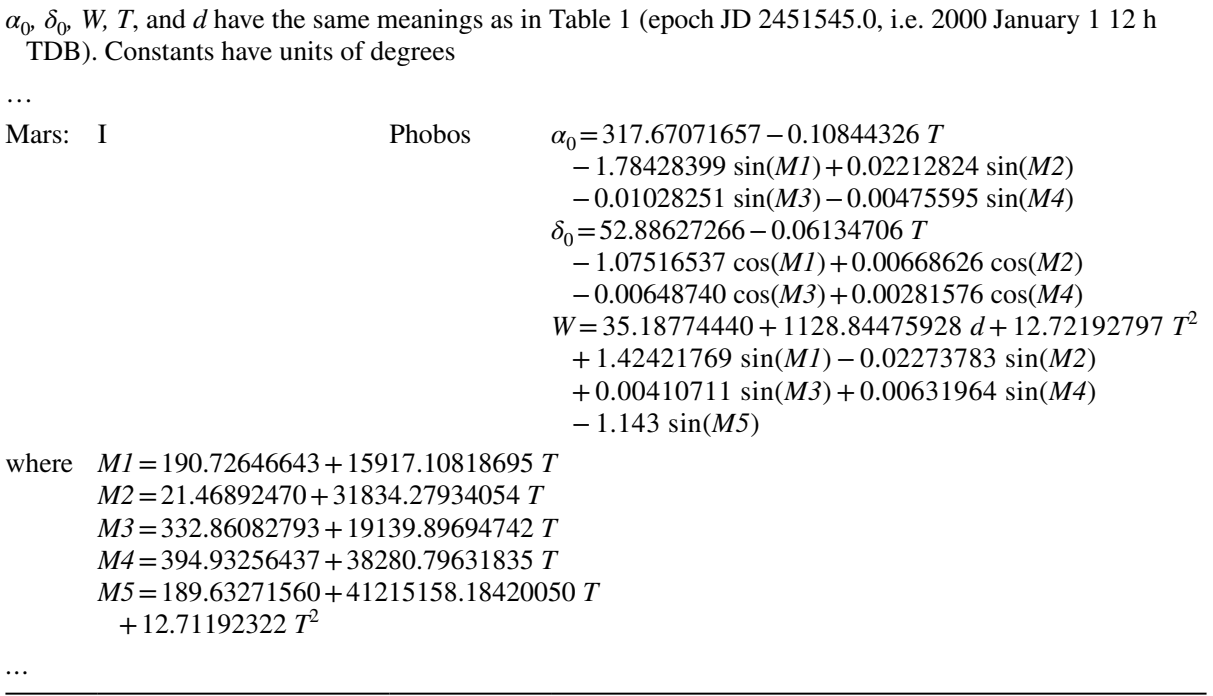

Also in this table (not shown) is a minor typographical error, the declination of the pole position for Deimos, instead of just " $\delta$ " should read " $\delta_{0}$ ".

The IAU Working Group on Cartographic Coordinates and Rotational Elements takes full responsibility for and regrets these errors.

Acknowledgements We thank Mike Garrahan and co-author Wang for bringing to our attention the sign error in Figs. 1 and 2. Likewise we thank co-authors Wang and Stark for bringing our attention to the errors in the prime meridian expression for Phobos. Archinal received support under a NASA-U. S. Geological Survey Interagency agreement.

\section{References}

Archinal, B.A., Acton, C.H., A'Hearn, M.F., Conrad, A., Consolmagno, G.J., Duxbury, T., Hestroffer, D., Hilton, J.L., Kirk, R., Klioner, S.A., McCarthy, D., Meech, K., Oberst, J., Ping, J., Seidelmann, P.K., Tholen, D.J., Thomas, P.C., Williams, I.P.: Report of the IAU working group on cartographic coordinates and rotational elements: 2015. Cel. Mech. Dyn. Ast. 130, 22 (2018). https://doi.org/10.1007/ s10569-017-9805-5

Burmeister, S., Willner, K., Schmidt, V., Oberst, J.: Determination of Phobos' rotational parameters by an inertial frame bundle block adjustment. J. Geodesy 92(9), 963-973 (2018). https://doi.org/10.1007/ s00190-018-1112-8

Stark, A. Willner, K., Burmeister, S., Oberst, J.: Geodetic framework for Martian satellite exploration I: reference rotation models. In: European Planetary Science Conference, vol. 11, EPSC2017-868-1 (2017). http://meetingorganizer.copernicus.org/EPSC2017/EPSC2017-868-1.pdf. Accessed 6 Sep 2019

Publisher's Note Springer Nature remains neutral with regard to jurisdictional claims in published maps and institutional affiliations. 